\title{
Searching for Photometric Variability across the L, T \& Y Dwarf Sequence
}

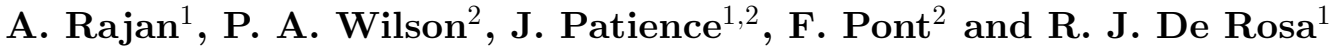 \\ ${ }^{1}$ School of Earth \& Space Exploration, Arizona State University, Tempe, AZ USA 85281 \\ email: arajan6@asu.edu \\ ${ }^{2}$ Astrophysics Group, School of Physics, University of Exeter, Exeter EX4 4QL, UK
}

\begin{abstract}
To investigate the atmospheres of ultracool brown dwarfs with temperatures covering the range of transiting and directly imaged planets, we have monitored a sample of $76 \mathrm{~L}$, T and Y brown dwarfs for infrared photometric variability. This survey was conducted in the $J$-band using both the SOFI camera on the 3.5-m NTT and the SWIRC camera on the 6.5-m MMT. Each target was observed for a period ranging from 2.0 hours to 6.0 hours, covering a significant fraction of the expected rotation period. Breakup of the iron and silicate clouds into a patchy cloud layer has been suggested as an explanation for the variability of several objects identified at the $\mathrm{L} / \mathrm{T}$ transition, and a similar process with sulfide clouds may be manifest in $\mathrm{T} / \mathrm{Y}$ transition objects; our data provides the first test of these patchy cloud scenarios across the entire brown dwarf spectral range.
\end{abstract}

Keywords. stars: low-mass, brown dwarfs, stars:variables:others, techniques: photometric

\section{Introduction}

With the discovery of Y-dwarfs by the WISE satellite (Wright et al. 2010), brown dwarfs now span the entire temperature range between the coolest stars and giant planets both in our Solar System and around other stars. Their low mass ensures that the core never undergoes nuclear fusion (e.g: Hayashi \& Nakano 1963), and so they cool throughout their lifetime resulting in an evolution of the chemical and physical properties. Theoretical models invoke the formation and dissipation of dusty condensate clouds to explain the measured colors and spectral features (e.g: Allard et al. 2001). Photometric variability monitoring provides a means to search for evidence of cloud features, storms, and activity in the atmospheres of these objects.

\section{Sample and Observations}

In two separate surveys conducted over the past two years we have observed objects spanning the entire L, T and Y spectral range. The first survey sample consists of $71 \mathrm{~L}$ and $\mathrm{T}$ brown dwarfs selected to provide an even sampling across the entire L-T spectral range (Wilson et al. 2013). Observations were taken with SOFI on the 3.5-m NTT over two separate epochs in 2011/12. The second survey consists of 5 late $\mathrm{T}$ and $\mathrm{Y}$ brown dwarfs that cover the $\mathrm{T} / \mathrm{Y}$ transition region (Rajan et al. 2013), where recent models predict the formation of sulfide clouds. The T-Y dataset was observed with SWIRC at the 6.5-m MMT over two nights in Mar 2012. Both surveys were carried out in the $J$ band where the variability is expected to be greatest. The targets were observed typically for 2-6 hours each and calibrated with a flat field, sky and dark frames. Aperture photometry was carried out using DAOPHOT and the light curves were corrected for airmass. 
Table 1. Variables identified in the both surveys

\begin{tabular}{llccc}
\hline \multicolumn{1}{c}{ Object } & Sp. Type & DOF & $\chi_{\text {red }}^{2}$ & Amplitude \\
\hline 2M0034 + 0523 & T6.5 (IR) & 7 & 2.1 & $2.2 \pm 0.8 \%$ \\
2M0136 + 0933 & T2.5 (IR) & 7 & 4.5 & $2.8 \pm 0.6 \%$ \\
2M0348 - 6022 & T7 (IR) & 7 & 6.7 & $2.2 \pm 0.8 \%$ \\
2M0445 - 3048 & L2 (Opt.) & 9 & 2.4 & $3.0 \pm 1.1 \%$ \\
W0458 + 6434 & T8.5 (IR) & 16 & 6.3 & $22.4 \pm 2.7 \%$ \\
2M0949 - 1545 & T2 (IR) & 6 & 4.1 & $6.5 \pm 1.4 \%$ \\
2M1010 - 0406 & L6 (Opt.) & 9 & 2.9 & $4.4 \pm 1.3 \%$ \\
2M1225 - 2739 & T6 (IR) & 7 & 3.7 & $3.6 \pm 1.3 \%$ \\
2M1828 - 4849 & T5.5 (IR) & 7 & 2.8 & $2.1 \pm 0.8 \%$ \\
2M2139 + 0220 & T1.5 (IR) & 6 & 12.8 & $4.9 \pm 1.1 \%$ \\
2M2228 - 4310 & T6.5 (IR) & 7 & 4.0 & $1.8 \pm 0.7 \%$ \\
2M2252 - 1730 & L7.5 (IR) & 6 & 1.4 & $1.7 \pm 1.0 \%$ \\
2M2356 - 1553 & T6 (IR) & 6 & 13.3 & $2.2 \pm 1.3 \%$ \\
\hline
\end{tabular}

Temperature Range (K)

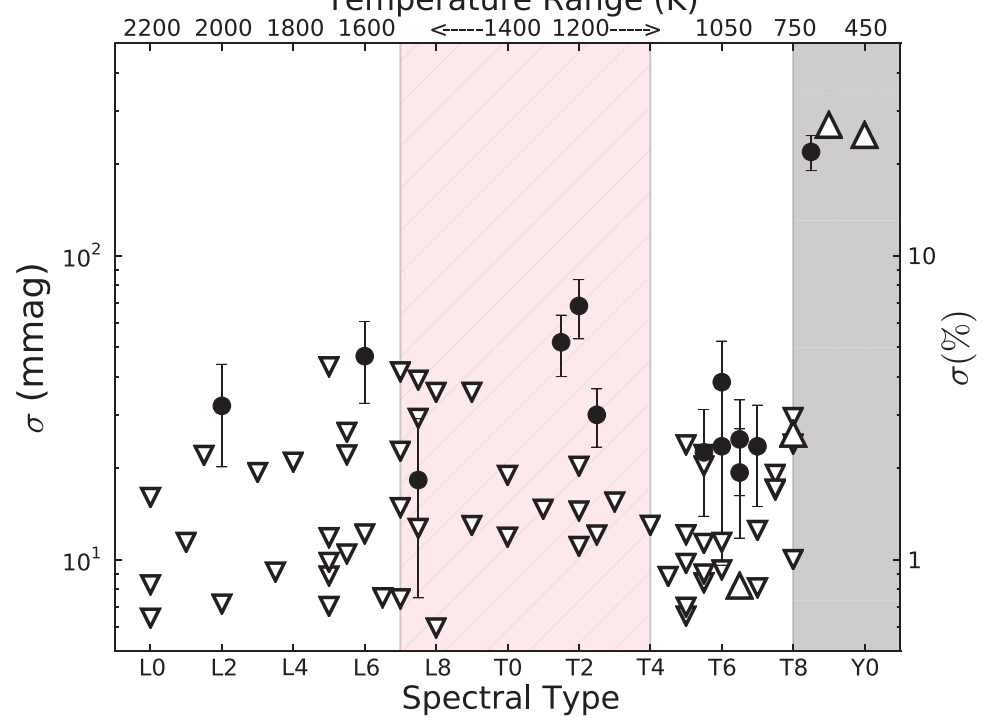

Figure 1. Preliminary results from the two surveys: open triangles indicate the limits and filled circles indicate confirmed variables. Shaded and hatched regions shows the transition regions.

\section{Results}

We detect 12 variables in the L-T sample (see Table 1) giving a variability fraction of $22_{-5}^{+7} \%$ for observations with a photometric quality better than $10 \mathrm{mmag}(1 \%)$, consistent with previous studies. We detect no correlation with spectral type and we also find that variability above the $5 \%$ level at $J$-band wavelengths is rare. One of the T-Y sample, which happens to be a binary system showed large variability at levels seen at the $\mathrm{L} / \mathrm{T}$ transition. Figure 1 shows the results for the entire L-T-Y survey.

\section{References}

Allard, F., Hauschildt, P. H., Alexander, D. R., et al. 2001, ApJ, 556, 357

Hayashi, C. \& Nakano, T. 1963, PThPh, 30, 460

Rajan, A., Patience, J., Wilson, P. A., Pont, F., \& De Rosa, R. J. 2013, A\&\&A, submitted

Wilson, P. A., Rajan, A., Patience, J., \& Pont, F. 2013, MNRAS, in prep

Wright, E. L., Eisenhardt, P. R. M., Mainzer, A. K., et al. 2010, AJ, 140, 1868 